\title{
Ground state of small mixed helium and spin-polarized tritium clusters: a quantum Monte Carlo study
}

P. Stipanović, ${ }^{1}$ L. Vranješ Markić, ${ }^{1,2, a)}$ J. Boronat, ${ }^{3}$ and B. Kežić ${ }^{1}$

1) Faculty of Science, University of Split, HR-21000 Split, Croatia

${ }^{2)}$ Institut für Theoretische Physik, Johannes Kepler Universität, A-4040 Linz, Austria

3) Departament de Física i Enginyeria Nuclear, Campus Nord B4-B5, Universitat Politècnica de Catalunya, E-08034 Barcelona, Spain

(Dated: 7 October 2018)

We report results for the ground-state energy and structural properties of small ${ }^{4} \mathrm{He}-$ $\mathrm{T} \downarrow$ clusters consisting of up to $4 \mathrm{~T} \downarrow$ and $8{ }^{4} \mathrm{He}$ atoms. These results have been obtained using very well-known ${ }^{4} \mathrm{He}-{ }^{4} \mathrm{He}$ and $\mathrm{T} \downarrow-\mathrm{T} \downarrow$ interaction potentials and several models for the ${ }^{4} \mathrm{He}-\mathrm{T} \downarrow$ interatomic potential. All the calculations have been performed with variational and diffusion Monte Carlo methods. It takes at least three atoms to form a mixed bound state. In particular, for small clusters the binding energies are significantly affected by the precise form of the ${ }^{4} \mathrm{He}-\mathrm{T} \downarrow$ interatomic potential but the stability limits remain unchanged. The only exception is the ${ }^{4} \mathrm{He}_{2} \mathrm{~T} \downarrow$ trimer whose stability in the case of the weakest ${ }^{4} \mathrm{He}-\mathrm{T} \downarrow$ interaction potential is uncertain, while it seems stable for other potentials. The mixed trimer ${ }^{4} \mathrm{He}(\mathrm{T} \downarrow)_{2}$, a candidate for Borromean state, is not bound. All other studied clusters are stable. Some of the weakest bound clusters can be classified as quantum halos, as a consequence of having high probability of being in a classically forbidden region.

PACS numbers: $67.65 .+z, 02.70 . S s$

a) Electronic mail: leandra@pmfst.hr 


\section{INTRODUCTION}

There are many similarities between spin-polarized tritium $(\mathrm{T} \downarrow)$ and ${ }^{4} \mathrm{He}$ atoms. Both are bosons, have a small mass and a weakly attractive interaction potential at large distances. Just like ${ }^{4} \mathrm{He}$, bulk $\mathrm{T} \downarrow$ remains liquid in the limit of zero temperature and zero pressure. In 1976, Stwaley and Nosanow suggested that $\mathrm{T} \downarrow$ should behave very much like liquid ${ }^{4} \mathrm{He}$ and therefore constitute a second example of bosonic superfluid. $\frac{1}{}$ Many similarities between the two systems have been confirmed by recent quantum Monte Carlo $\underline{2}$ results. For instance, bulk $\mathrm{T} \downarrow$ is a superfluid at zero temperature, with a condensate fraction $n_{0}=0.129(3)$ at the equilibrium density. Miller examined the bulk ${ }^{4} \mathrm{He}-\mathrm{T} \downarrow$ system as an example of binary mixtures using a variational ansatz and found that it prefers to be completely phaseseparated $\underline{\underline{3}}$

${ }^{4} \mathrm{He}$ clusters have been extensively studied, both theoretically and experimentally, exhibiting nanoscopic superfluidity. Due to the similarities between ${ }^{4} \mathrm{He}$ and $\mathrm{T} \downarrow$, a similar behavior of spin-polarized tritium clusters is expected. So far, $\mathrm{T} \downarrow$ clusters have been investigated only theoretically. Small clusters of $\mathrm{T} \downarrow$ were for the first time studied by Blume et al. $\underline{4}$. The smallest bound $\mathrm{T} \downarrow$ cluster is the trimer, showing characteristics of a Borromean or halo state. $\frac{5 \cdot 6}{6}$ Its tiny binding energy of only around $4 \mathrm{mK}$ has been later confirmed by other authors $\mathbf{z}^{\underline{7} .8}$. The extension of the study to larger clusters ${ }^{9}$ confirmed that $\mathrm{T} \downarrow$ clusters are much more weakly bound and diffuse than the ${ }^{4} \mathrm{He}$ clusters with the same number of atoms. The stability of mixed clusters of $T \downarrow \downarrow \underline{\underline{8}} \underline{10}$ with spin-polarized hydrogen $(\mathrm{H} \downarrow)$ and spin-polarized deuterium $(\mathrm{D} \downarrow)$, in particular small ones, has also been investigated. For the clusters with $\mathrm{D} \downarrow$ the stability limits depend on the number of $\mathrm{D} \downarrow$ atoms and the occupation of its nuclear spin states. On the other hand, due to the small mass of $\mathrm{H} \downarrow$, it has been shown that even $60 \mathrm{~T} \downarrow$ atoms are not enough to bind one $\mathrm{H} \downarrow$ atom.

Mixed ${ }^{4} \mathrm{He}-\mathrm{T} \downarrow$ clusters have not been studied yet. $\mathrm{T} \downarrow$ has almost the same mass as ${ }^{3} \mathrm{He}$, but it is a boson, so it will exhibit different physical properties. Because of the small mass of both ${ }^{4} \mathrm{He}$ and $\mathrm{T} \downarrow$ and weak attractive parts of the interaction potentials it is expected that the smallest clusters could be extremely weakly bound. If ${ }^{4} \mathrm{He}(\mathrm{T} \downarrow)_{2}$ is bound, it would be an example of yet another Borromean state, because none of its subsystems are bound. The only mixed molecular system for which Borromean state has been predicted theoretically so

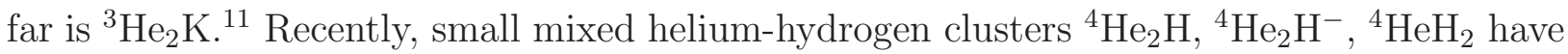


been studied as well. $12-16$

Weakly bound clusters are also candidates for Efimov states,$\frac{17}{1}$ which are predicted to exist also in mixed systems. Interest in these states and other universal binding properties of small clusters has been significantly intensified in the last couple of years by the research in the field of cold gases. After the experimental detection of an Efimov state in an ultracold gas of cesium atoms $\stackrel{18}{\underline{10}}$ giant Efimov trimers have been detected in other systems $\underline{19}-21$ including those of mixed species..$^{22}$ Later work was devoted to the study of the Efimov spectrum ${ }^{23}$ as well as to the prediction ${ }^{24}$ and detection ${ }^{25}$ of giant tetramers. Furthermore, a recent study of weakly bound bosonic clusters has found a series of universal cluster states, that can be qualitatively interpreted by adding one particle at a time to an Efimov trimer ${ }^{26}$

Although the physics of molecular weakly bound clusters is not the same as the one of ultracold gases, it is interesting to investigate if similar behavior of few-body states appear, indicating the universality. Universal physics is also connected to physics of quantum ha$\operatorname{los}, \underset{27-29}{2}$ defined as bound states of clusters of particles with a radius extending well into the classically forbidden region. It is therefore worth studying which small molecular clusters qualify as quantum halos.

In this work, we report the ground-state energy of clusters having up to $8{ }^{4} \mathrm{He}$ and 4 $\mathrm{T} \downarrow$ atoms, as well as their structural properties, obtained with the diffusion Monte Carlo (DMC) method. In Sec. II, we report briefly the DMC method and discuss the trial wave functions used for importance sampling. Sec. III reports the results obtained by the DMC simulations. Finally, Sec. IV comprises a summary of the work and an account of the main conclusions.

\section{METHOD}

\section{A. Interaction potentials}

We have modeled the ${ }^{4} \mathrm{He}-{ }^{4} \mathrm{He}$ interactions with the Aziz HFD-B(He) interaction potential ${ }^{30}$. Some calculations have also been performed with the Korona et al. SAPT potential. $\underline{31}^{3}$

The interatomic interaction between tritium atoms is described with the spin-independent central triplet pair potential $b^{3} \Sigma_{u}^{+}$, which was determined in an essentially exact way by Ko-

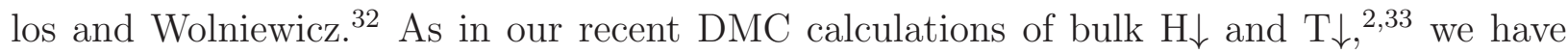


used the recent extension of Kolos and Wolniewicz data to larger interparticle distances by Jamieson et al. (JDW) $\stackrel{34}{\underline{34}}$ The potential is finally constructed using a cubic spline interpolation of JDW data, which is smoothly connected to the long-range behavior of the $T \downarrow-T \downarrow$ potential as calculated by Yan et al $\underline{\underline{35}}$ The JDW potential used in the present work has a core diameter $\sigma=3.67 \AA$ and a minimum of $-6.49 \mathrm{~K}$ at a distance $4.14 \AA$. We have previously verified that the addition of mass-dependent adiabatic corrections (as calculated by Kolos and Rychlewski ${ }^{36}$ ) to the JDW potential does not change the energy of bulk spinpolarized tritium. $\underline{\underline{2}}$ It is worth mentioning that within the Born-Oppenheimer approximation it has been explicitly shown that in the spin-aligned electronic state, tritium nuclei behave as effective bosons $\underline{\underline{37}}$

Several forms of ${ }^{4} \mathrm{He}-\mathrm{T} \downarrow$ interaction potentials are available in the literature, including those resulting from ab initio calculations and semiempirical potentials. In 1984, Jochemsen et al. $\underline{\underline{38}}$ proposed a semiempirical potential (R2) after discussing available potentials until that time and comparing theoretical data with diffusion experiments at low temperature. That potential is essentially the same as an older potential by Das et al $\underline{\underline{39}}$ (DWW), which the authors of Ref. 38 themselves preferred to use. The most sophisticated ab initio calculation is that of Meyer and Frommhold from 1994 (MF), who obtained good agreement with experiment, except at very low temperatures $\underline{\underline{40}}$ Subsequently, Chung and Dalgarno $\underline{\underline{41}}$ introduced slight modifications in the short-range repulsive part of the MF potential that resulted in a better agreement with diffusion measurements at low temperatures (MFmod). In both MF and MFmod potentials, the construction of the potential-energy curve at large separations and the dispersion component that determines the long-range behavior in MF and MFmod is of high precision. $\frac{35}{}$ All of our calculations have been performed with both the DWW and the MFmod potential. In addition, for some selected clusters we have performed calculations using some other forms of available potentials. Specifically, Toennies et al $\underline{\underline{42}}$ have proposed the Lennard-Jones 6-12 potential (TWW) whose parameters have been determined using data from low energy elastic scattering. TWW has the largest core, smallest depth and the strongest long-range part of all the studied potentials. Due to its simplicity it has been used also in some very recent calculations, e.g. by Krotscheck and Zillich; $\underline{\underline{43}}$ these authors have found that, in the bulk liquid, the binding energies obtained with TWW differ less than $5 \%$ from those that are obtained with the DWW potential. Finally, we include the MF potential and the potential by Tang and Yang $\underline{44}$ (TY), who used the Tang-Toennies 


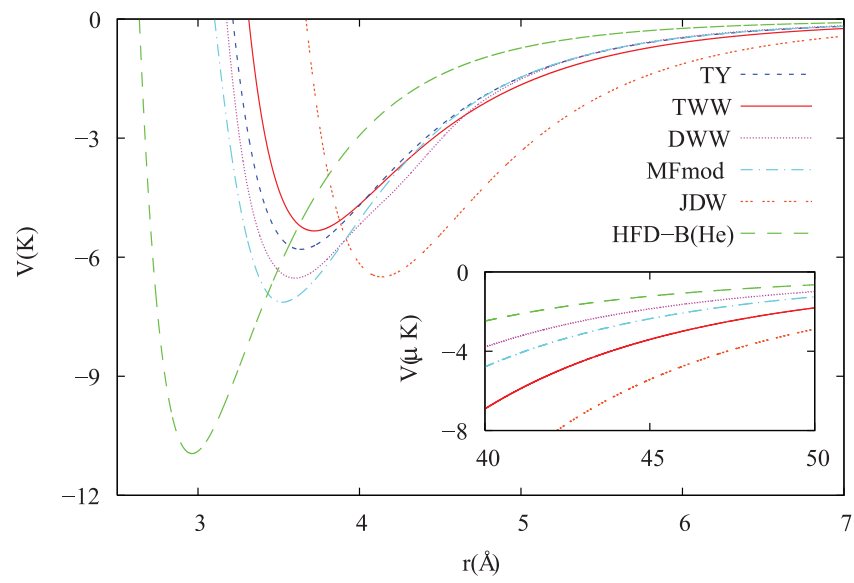

FIG. 1. Comparison among interaction potentials between ${ }^{4} \mathrm{He}-{ }^{4} \mathrm{He}\left(\mathrm{HFD}-\mathrm{B}(\mathrm{He}){ }^{30}\right),{ }^{4} \mathrm{He}-\mathrm{T} \downarrow(\mathrm{TY} \underline{44}$, TWW $\underline{42}$, DWW $\left.\underline{39}, M^{2} \bmod ^{40} \underline{41}\right)$ and $\mathrm{T}_{\downarrow}-\mathrm{T} \downarrow\left(\mathrm{JDW}^{34}\right)$. For long distances, TY and MFmod have basically the same behavior.

model. The comparison of these different potentials is presented in Figure 1. The strongest MFmod potential has a core diameter of $\sigma=3.10 \AA$ and a minimum of $-7.14 \mathrm{~K}$ at a distance $3.52 \AA$. The MF potential would be the same in the scale of the figure because the only differences are in the repulsive part. The DWW interaction has a core diameter of $\sigma=3.18 \AA$ and a minimum of $-6.53 \mathrm{~K}$ at a distance $3.60 \AA$, while the weakest TWW potential has a core diameter of $\sigma=3.31 \AA$ and a minimum of $-5.34 \mathrm{~K}$ at a distance $3.72 \AA$. In Fig. 1 the ${ }^{4} \mathrm{He}-{ }^{4} \mathrm{He}$ and $\mathrm{T} \downarrow-\mathrm{T} \downarrow$ interaction potentials have been included for comparison as well. Of all the interaction potentials, the $\mathrm{T} \downarrow-\mathrm{T} \downarrow$ one has the largest core and the most attractive long-range part.

\section{B. Diffusion Monte Carlo method}

The ground-state properties of the clusters have been studied using the DMC method, whose starting point is the many-body Schrödinger equation written in imaginary time,

$$
-\hbar \frac{\partial \Psi(\boldsymbol{R}, t)}{\partial t}=\left(H-E_{\mathrm{r}}\right) \Psi(\boldsymbol{R}, t)
$$

where $E_{\mathrm{r}}$ is a constant acting as a reference energy and $\boldsymbol{R} \equiv\left(\boldsymbol{r}_{1}, \ldots, \boldsymbol{r}_{N}\right)$ collectively denotes particle positions. 
The $N$-particle Hamiltonian $H$ for the cluster ${ }^{4} \mathrm{He}_{n}(\mathrm{~T} \downarrow)_{m}, n+m=N$ is

$$
H=-\sum_{i=1}^{N} \frac{\hbar^{2}}{2 m_{i}} \nabla_{i}^{2}+\sum_{\substack{i, j=1 \\ i<j}}^{n} V_{H e H e}\left(r_{i j}\right)+\sum_{i=1}^{n} \sum_{j=n+1}^{n+m} V_{H e T}\left(r_{i j}\right)+\sum_{\substack{i, j=n+1 \\ i<j}}^{n+m} V_{T T}\left(r_{i j}\right)
$$

where $V_{\text {HeHe }}(r), V_{H e T}(r)$ and $V_{T T}(r)$ are the interaction potentials between the different components of the mixture.

In DMC, the Schrödinger equation (11) is solved stochastically by multiplying $\Psi(\boldsymbol{R}, t)$ with $\psi(\boldsymbol{R})$, a trial wave function used for importance sampling, and rewriting Eq. (11) in terms of the mixed distribution $\Phi(\boldsymbol{R}, t)=\Psi(\boldsymbol{R}, t) \psi(\boldsymbol{R})$. Within the Monte Carlo framework, $\Phi(\boldsymbol{R}, t)$ is represented by a set of walkers $\boldsymbol{R}_{\boldsymbol{i}}$. In the limit $t \rightarrow \infty$, only the lowest energy eigenfunction, not orthogonal to $\psi(\boldsymbol{R})$, survives and then the sampling of the ground state is effectively achieved. Apart from statistical uncertainties, the energy of a $N$-body bosonic system is exactly calculated.

In the present simulations, the trial wave function has been written as a product of Jastrow two-body correlation functions between all the pairs,

$$
\psi_{\mathrm{J}}(\boldsymbol{R})=\prod_{\substack{i, j=1 \\ i<j}}^{n} f_{H e H e}\left(r_{i j}\right) \prod_{\substack{i, j=n+1 \\ i<j}}^{n+m} f_{T T}\left(r_{i j}\right) \prod_{i=1}^{n} \prod_{j=n+1}^{n+m} f_{H e T}\left(r_{i j}\right),
$$

where $f_{\mathrm{HeHe}}$ describes two-body correlations between helium atoms, $f_{T T}$ describes two-body correlations between spin-polarized tritium atoms and $f_{H e T}$ accounts for the ${ }^{4} \mathrm{He}-\mathrm{T} \downarrow$ pairs. Different types of two-body correlation function $f(r)$ have been tested,

$$
\begin{aligned}
& f_{1}(r)=\exp \left[-\left(\frac{b}{r}\right)^{5}-s r\right], \\
& f_{2}(r)=\exp \left[-\left(\frac{\alpha}{r}\right)^{\gamma}-s_{1} r\right] / r \\
& f_{3}(r)=\exp \left[-b_{1} \exp \left(-b_{2} r\right)-s_{2} r\right]
\end{aligned}
$$

where $b, s, \alpha, \gamma, s_{1}, b_{1}, b_{2}$ and $s_{2}$ are variational parameters. The optimization of the trial wave functions has been done for all clusters by means of the variational Monte Carlo (VMC) method. For clusters having less than 5 atoms it has been very difficult to obtain good quality VMC results so different forms have been tried out to ascertain that DMC gives the same value of the energy for different guiding wave functions. 
For ${ }^{4} \mathrm{He}-{ }^{4} \mathrm{He}$ correlations, a set of calculations for all clusters has been done using $f_{1}(r)$ (4), with the parameter $b=2.6 \AA$ and $s$ from 0.08 to $0.13 \AA^{-1}$. For some smaller clusters, $f_{2}(r)$ (5) has also been used, with optimal parameters in the range $\alpha \in[2.75,2.82] \AA$, $\gamma \in[4.1,4.7], s_{1} \in[0.001,0.015] \AA^{-1}$. For $\mathrm{T} \downarrow-\mathrm{T} \downarrow$ two-body correlations, the form $f_{3}(r)$ (6) has been chosen for all the clusters, based on the previous experience with pure and mixed $\mathrm{T} \downarrow$ clusters. $\underline{\underline{8}}$ The optimal parameters have been obtained in the range: $b_{1}$ from 82 to $94, b_{2}$ from 1.23 to $1.24 \AA^{-1}$, and $s_{2}$ from 0.04 to $0.06 \AA^{-1}$, where smaller values of $s$ correspond to larger clusters. The only exceptions are the clusters ${ }^{4} \mathrm{He}_{2}(\mathrm{~T} \downarrow)_{2}$ and ${ }^{4} \mathrm{He}(\mathrm{T} \downarrow)_{3,4}$ for which better energies were obtained with $b_{1}=200, b_{2}=1.5 \AA^{-1}$ and $s_{2}=0.065 \AA^{-1}$. In addition, for some of the smallest clusters variational calculations have also been done with the form $f_{1}(r)$ (44), where optimal parameters have been found around $\alpha=4.2 \AA, \gamma \in[3.15,3.82]$ and $s_{1} \approx 0.001 \AA^{-1}$.

The optimization of the ${ }^{4} \mathrm{He}-\mathrm{T} \downarrow$ correlations has been the most demanding: it has been done for several potentials and forms of trial wave functions. First, for the DWW potential, $f_{1}(r)$ has been used with $b$ around $3 \AA$ and $s$ ranging from 0.04 to $0.06 \AA^{-1}$. With $f_{1}(r)$ and clusters with more than 5 atoms the VMC energies approach the $80 \%$ of the DMC values. Therefore, for the smallest clusters, $f_{2}(r)$ has been tried as well, with resulting parameters $\alpha \in[3.59,3.69] \AA, \gamma \in[3.08,3.20], s_{1}=0.001 \AA^{-1}$. In conclusion, VMC energies reached more than $40 \%$ of the DMC ones in all cases except ${ }^{4} \mathrm{He}_{2} \mathrm{~T} \downarrow$, where negative energy could not be obtained at the VMC level for the DWW potential. For this cluster, the best set of parameters for the function $f_{2}(r)$ is $\alpha=3.62 \AA, \gamma=3.1, s_{1}=0.001 \AA^{-1}$, although it arrives to the same DMC value for other sets of parameters. In addition, ${ }^{4} \mathrm{He}-\mathrm{T} \downarrow$ correlations for that cluster have also been modeled with $f_{3}(r)(\underline{6})\left(b_{1}=300, b_{2}=1.93 \AA^{-1}\right.$ and $s_{2}=0.05$ $\AA^{-1}$ ) and despite significant differences at the VMC level, DMC has arrived to the same value of the energy. Variational parameters differ only slightly for other types of ${ }^{4} \mathrm{He}-\mathrm{T} \downarrow$ interaction potentials, except for trimers and tetramers, where the differences are somewhat larger.

We have verified that 1000 walkers are enough for excluding the bias coming from the size of the population ensemble used in a simulation. The same conclusion emerged also from previous experience in pure and mixed $\mathrm{T} \downarrow$ clusters. The only exception is again the ${ }^{4} \mathrm{He}_{2} \mathrm{~T} \downarrow$ cluster, for which a larger number of walkers has been necessary: 2000 for the DWW potential and 10000 for the TWW potential. 
In order to eliminate bias coming from the time-step value used in simulations, all calculations have been performed with several time-steps $(\Delta t)$ which assume values within the interval $3 \times 10^{-4}-5 \times 10^{-3} \mathrm{~K}^{-1}$, where minimum and maximum values in that interval have been adjusted depending on the size of the cluster. The ground-state energies for different time-steps have then been extrapolated to $\Delta t \rightarrow 0$. In accordance with the DMC method used in this work, which is accurate to second order in the time step, $\underline{45}$ the extrapolation is made with a quadratic function.

The potential energies and distribution functions have been obtained using pure estimators. $\underline{46}^{-}$The asymptotic block size for each cluster and type of ${ }^{4} \mathrm{He}-\mathrm{T} \downarrow$ interaction potential has been determined from the behavior of the potential energy versus the block size. For most of the clusters, 2500 steps per block have been enough to arrive to the asymptotic value of the potential energy. In the case of smaller clusters, having less than five atoms, the block size grows to 10000-20000 steps reflecting the low quality of the trial wave functions. Even 50000 steps in the block have been needed for the TWW potential. However, for blocks of this size the same values of pure estimators for different types of trial wave functions have been obtained and therefore DMC estimations of these magnitudes are unbiased.

\section{RESULTS}

Ground-state energies of all the studied clusters for two types of ${ }^{4} \mathrm{He}-\mathrm{T} \downarrow$ interaction potentials are presented in Table [1. Energy in absolute value as a function of the number of ${ }^{4} \mathrm{He}$ and $\mathrm{T} \downarrow$ atoms is presented in Fig. 2 for the DWW interaction potential. In addition, calculations which have been made for several selected clusters with other types of potentials are presented in Table

As shown in Table I, it takes at least three atoms to form a mixed bound state. The weakest bound cluster is ${ }^{4} \mathrm{He}_{2} \mathrm{~T} \downarrow$. However, the value of its binding energy depends strongly on the type of the interaction potential. For the DWW, MF and MFmod potentials, it is lower than the energy of the ${ }^{4} \mathrm{He}_{2}$ dimer, which is $-1.66 \mathrm{mK}$, as calculated in Ref. 47. For the two weaker potentials (TWW, TY) the obtained energy is within the errorbar equal to the energy of the ${ }^{4} \mathrm{He}_{2}$ dimer. Thus, in the case of the latter two potentials it is unclear whether this cluster is stable with respect to the separation in the ${ }^{4} \mathrm{He}_{2}$ dimer and a free $\mathrm{T} \downarrow$ atom. On the other hand, for the potentials in which ${ }^{4} \mathrm{He}_{2} \mathrm{~T} \downarrow$ is surely bound the 
TABLE I. Ground-state energies (in $\mathrm{mK}$ ) of the studied ${ }^{4} \mathrm{He}-\mathrm{T} \downarrow$ clusters. In each cell top result and bottom result are obtained using the DWW $\underline{39}$ and MFmod ${ }^{41}{ }^{4} \mathrm{He}-\mathrm{T} \downarrow$ interactions, respectively.

\begin{tabular}{|c|c|c|c|c|}
\hline & 1 & 2 & 3 & 4 \\
\hline \multirow{2}{*}{1} & & & $-56(3)$ & $-285(5)$ \\
\hline & & & $-92(5)$ & $-355(5)$ \\
\hline \multirow{2}{*}{2} & $-3.0(2)$ & $-80(6)$ & $-284(6)$ & $-635(6)$ \\
\hline & $-16.6(8)$ & $-135(9)$ & $-406(8)$ & $-819(9)$ \\
\hline \multirow{2}{*}{3} & $-235(10)$ & $-444(5)$ & $-790(10)$ & $-1248(10)$ \\
\hline & $-290(16)$ & $-577(5)$ & $-1007(6)$ & $-1552(6)$ \\
\hline \multirow{2}{*}{4} & $-790(5)$ & $-1120(6)$ & $-1577(5)$ & $-2145(11)$ \\
\hline & $-883(4)$ & $-1318(4)$ & $-1885(4)$ & $-2560(6)$ \\
\hline \multirow{2}{*}{5} & $-1664(5)$ & $-2105(6)$ & $-2640(6)$ & $-3310(14)$ \\
\hline & $-1784(5)$ & $-2356(8)$ & $-3014(7)$ & $-3825(8)$ \\
\hline \multirow{2}{*}{6} & $-2785(7)$ & $-3335(9)$ & $-3975(6)$ & $-4715(13)$ \\
\hline & $-2925(8)$ & $-3636(8)$ & $-4433(10)$ & $-5320(8)$ \\
\hline \multirow{2}{*}{7} & $-4145(11)$ & $-4785(7)$ & $-5510(7)$ & $-6330(13)$ \\
\hline & $-4302(6)$ & $-5121(8)$ & $-6008(10)$ & $-7015(8)$ \\
\hline \multirow{2}{*}{8} & $-5725(8)$ & $-6455(11)$ & $-7250(10)$ & $-8155(6)$ \\
\hline & $-5908(8)$ & $-6834(12)$ & $-7818(10)$ & $-8916(14)$ \\
\hline
\end{tabular}

value of the binding energy varies from -3.0(2) $\mathrm{mK}$ to $-16.6(8) \mathrm{mK}$. Such small energies result from a huge cancellation between kinetic and potential energies. For instance, in case of the DWW interaction potential the potential energy of the ${ }^{4} \mathrm{He}_{2} \mathrm{~T} \downarrow$ is $E_{p}=-300(20)$ $\mathrm{mK}$, as determined using pure estimators. The kinetic energy, determined as a difference between the total and potential energy is then $297(20) \mathrm{mK}$. A similar system, ${ }^{4} \mathrm{He}_{2} \mathrm{H}$ has recently been investigated using an interaction potential which is in between the DWW and MFmod $\stackrel{48}{ }$ and no bound states have been found. 12,15,16 This is very much in agreement with the present result, since the three times lower mass of hydrogen increases the kinetic energy of the system, which already for tritium almost cancels the potential energy. In Ref. 12 other isotopic combinations have been studied as well using hyperspherical coordinates in 


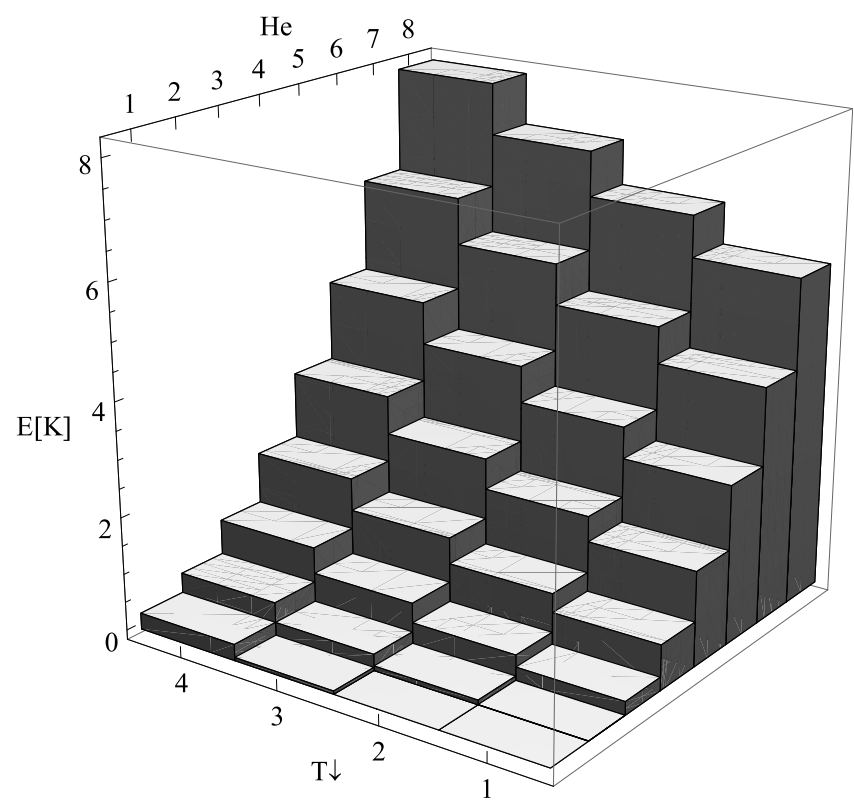

FIG. 2. The absolute values of the ground-state energies are presented as a function of the number of ${ }^{4} \mathrm{He}$ and $\mathrm{T} \downarrow$ atoms, when using the DWW ${ }^{39}$ potential to model the ${ }^{4} \mathrm{He}-\mathrm{T} \downarrow$ interaction.

TABLE II. Ground-state energies (in $\mathrm{mK}$ ) of investigated ${ }^{4} \mathrm{He}-\mathrm{T} \downarrow$ clusters.

\begin{tabular}{c|c|c|c|c}
\hline \hline \multicolumn{2}{c|}{ Cluster } & \multicolumn{3}{|c}{${ }^{4} \mathrm{He}-\mathrm{T} \downarrow$ interaction potential } \\
\hline${ }^{4} \mathrm{He}$ & $\mathrm{T} \downarrow$ & $\mathrm{TWW}$ & $\mathrm{TY}$ & $\mathrm{MF}$ \\
\hline 2 & 1 & $-1.3(3)$ & $-1.4(4)$ & $-13.9(8)$ \\
3 & 2 & $-254(12)$ & $-288(15)$ & $-522(4)$ \\
3 & 4 & $-781(11)$ & $-877(7)$ & $-1434(8)$ \\
4 & 2 & $-834(6)$ & $-886(6)$ & $-1243(5)$ \\
4 & 4 & $-1503(10)$ & $-1633(7)$ & $-2402(10)$ \\
6 & 1 & $-2579(6)$ & $-2605(7)$ & $-2863(9)$ \\
6 & 4 & $-3826(10)$ & $-3990(10)$ & $-5075(11)$ \\
\hline \hline
\end{tabular}

the adiabatic approximation. One bound state has been found for ${ }^{4} \mathrm{He}_{2} \mathrm{~T}$ system, with the energy of $-6.8 \mathrm{mK}$. Since the authors do not use the same form of the ${ }^{4} \mathrm{He}-{ }^{4} \mathrm{He}$ interaction potential it is not possible to directly compare this value with our results; however we obtain qualitative agreement.

An extensive search for a bound state of the other mixed trimer ${ }^{4} \mathrm{He}(\mathrm{T} \downarrow)_{2}$, a possible 
Borromean state, has been performed. However, for all of the studied potentials and different types of trial wave functions, the energies in the DMC calculations remained positive. Moreover, in the course of the simulation, particles were going more and more away from each other. This case can be compared with the ${ }^{4} \mathrm{He}^{3} \mathrm{He}_{2}$ cluster which has basically the same mass, but interacts with a stronger potential and is likewise not bound. $\underline{49}$

All the other studied mixed clusters with up to four $\mathrm{T} \downarrow$ and eight ${ }^{4} \mathrm{He}$ atoms are bound. In absolute value, the energy grows with the addition of both ${ }^{4} \mathrm{He}$ and $\mathrm{T} \downarrow$ atoms, but it takes about two $\mathrm{T} \downarrow$ atoms to achieve the same increase in binding as with the addition of one ${ }^{4} \mathrm{He}$ atom, as can be seen in Fig. 2. Significant cancellation between kinetic and potential energies persists for almost all clusters, with total energies being $1 \%$ to $20 \%$ of the potential energy for the largest cluster. The differences in the binding energies for different potentials are lowered with the increase of the cluster size and in particular with combined lowering of the fraction of $\mathrm{T} \downarrow$ atoms in it. This behavior can be more easily seen in Fig. 3 which shows the difference between ground-state energies in calculations with MFmod and DWW potentials, divided by the ground-state energy with the MFmod potential $\left(E_{M F m o d}-E_{D a s}\right) / E_{M F m o d}$, as a function of the number of ${ }^{4} \mathrm{He}$ and $\mathrm{T} \downarrow$ atoms in the cluster. Qualitatively, the stability of the clusters, except for ${ }^{4} \mathrm{He}_{2} \mathrm{~T} \downarrow$, is not sensitive to the forms of the interaction potentials. However, the relationship of binding strengths between different clusters is not always the same for different potentials. For example, ${ }^{4} \mathrm{He}_{7}(\mathrm{~T} \downarrow)_{2}$ is more strongly bound than ${ }^{4} \mathrm{He}_{6}(\mathrm{~T} \downarrow)_{4}$ for the DWW potential, while for the MFmod one this relationship is reversed. Some calculations have been performed using another form of the ${ }^{4} \mathrm{He}-{ }^{4} \mathrm{He}$ interaction potential ${ }^{31}$. The resulting energies are basically equal within the errorbars. For example, for ${ }^{4} \mathrm{He}_{2} \mathrm{~T} \downarrow$ with the HFD-B(He) potential and DWW one obtains -3.0(2) mK, and with SAPT 31 and DWW -3.3(2) mK. The ground-state energy of the larger cluster ${ }^{4} \mathrm{He}_{8} \mathrm{~T} \downarrow$ is $-5770(10) \mathrm{mK}$ with SAPT, and $-5725(8) \mathrm{mK}$ with HFD-B(He).

The smaller clusters are especially interesting. For example, ${ }^{4} \mathrm{He}(\mathrm{T} \downarrow)_{3,4}$ has no bound two-particle subsystem, while out of all six two-particle interactions in ${ }^{4} \mathrm{He}_{2}(\mathrm{~T} \downarrow)_{2}$ only the one between two ${ }^{4} \mathrm{He}$ atoms would give a bound subsystem. The latter cluster is similar to ${ }^{4} \mathrm{He}_{2}{ }^{3} \mathrm{He}_{2}$, which authors of Ref. 50 have called pseudo-Borromean state because of the fact that it has also only one bound subsystem.

The comparison between ${ }^{4} \mathrm{He}_{n}(\mathrm{~T} \downarrow)_{m}$ and ${ }^{4} \mathrm{He}_{n}{ }^{3} \mathrm{He}_{m}$ is interesting because both types of clusters have essentially the same mass, but differ in the interaction potentials and the statis- 


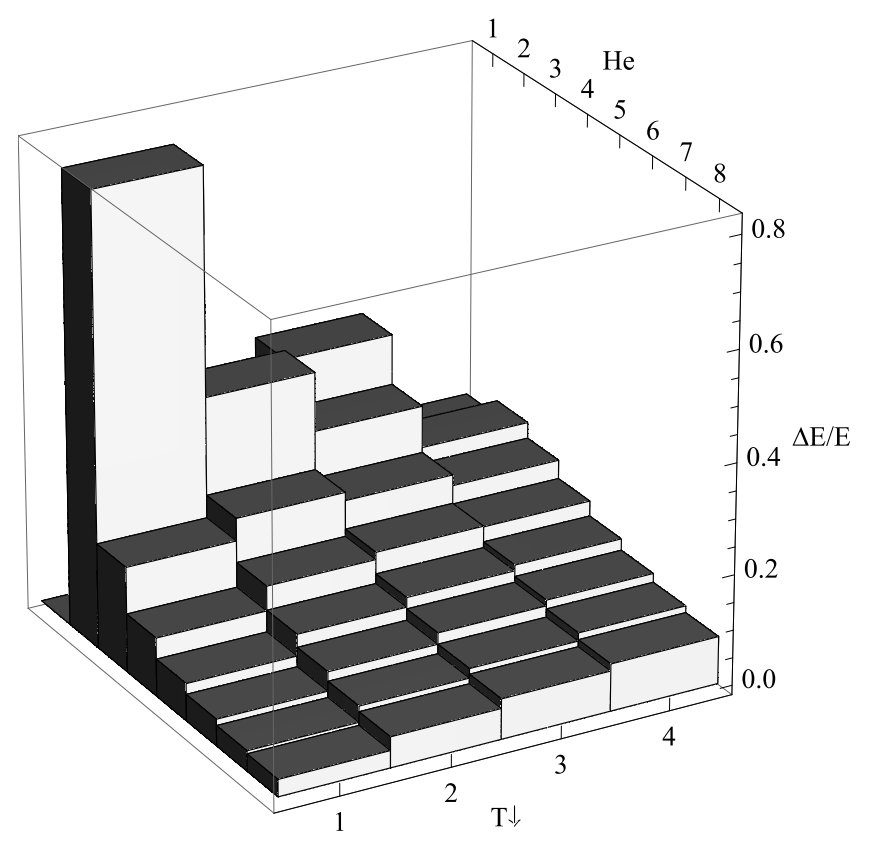

FIG. 3. The difference between ground-state energies in calculations with the MFmod $\underline{41}$ and DWW $\underline{\underline{39}}$ potentials, divided by the ground-state energy with the MFmod potential, $\Delta E / E=$ $\left(E_{M F m o d}-E_{D a s}\right) / E_{M F m o d}$, as a function of the number of ${ }^{4} \mathrm{He}$ and $\mathrm{T} \downarrow$ atoms in the cluster.

tics. The interaction potential between two helium atoms is the same for all combinations of isotopes and is stronger than both the ${ }^{4} \mathrm{He}-\mathrm{T} \downarrow$ and $\mathrm{T} \downarrow-\mathrm{T} \downarrow$ one. Thus, as expected, the clusters ${ }^{4} \mathrm{He}_{n}{ }^{3} \mathrm{He}_{m}$ are more strongly bound than ${ }^{4} \mathrm{He}_{n}(\mathrm{~T} \downarrow)_{m}$ for $m=1,2$. However, for more than two ${ }^{3} \mathrm{He}$ atoms the fermion statistics comes into play. Thus for example ${ }^{4} \mathrm{He}^{3} \mathrm{He}_{3,4}$ and ${ }^{4} \mathrm{He}_{2}{ }^{3} \mathrm{He}_{3,4}$ are unbound, but the clusters ${ }^{4} \mathrm{He}(\mathrm{T} \downarrow)_{3,4}$ and ${ }^{4} \mathrm{He}_{2}(\mathrm{~T} \downarrow)_{3,4}$ have bound states. For larger clusters, despite the fermionic nature of ${ }^{3} \mathrm{He},{ }^{4} \mathrm{He}_{n}{ }^{3} \mathrm{He}_{m}$ show tendency to be more strongly bound than ${ }^{4} \mathrm{He}_{n}(\mathrm{~T} \downarrow)_{m}$, for increasing $n$, as can be seen in Fig. 4 for the DWW potential, but is not yet clear for the MFmod. The precise $m$ for which this transition occurs can not be predicted because the energies of ${ }^{4} \mathrm{He}_{n}{ }^{3} \mathrm{He}_{m}$ are not calculated for all $n$ and $m$ with the HFD-B(He) interaction potential.

In addition to energy, the structure has also been determined by calculating with pure estimators the distribution of particles to the centre of mass of the system $\rho(r)$ and the distribution of the separations between particles $P(r)$. These functions have been normalized as $\int \rho(r) d^{3} r=N$, where $N$ is the number of particles of particular species in the cluster and $\int P(r) d r=1$. Both functions, for the smallest cluster ${ }^{4} \mathrm{He}_{2} \mathrm{~T} \downarrow$ are shown in Fig 5 . The larger plot shows huge differences between distributions of particle separations for different 


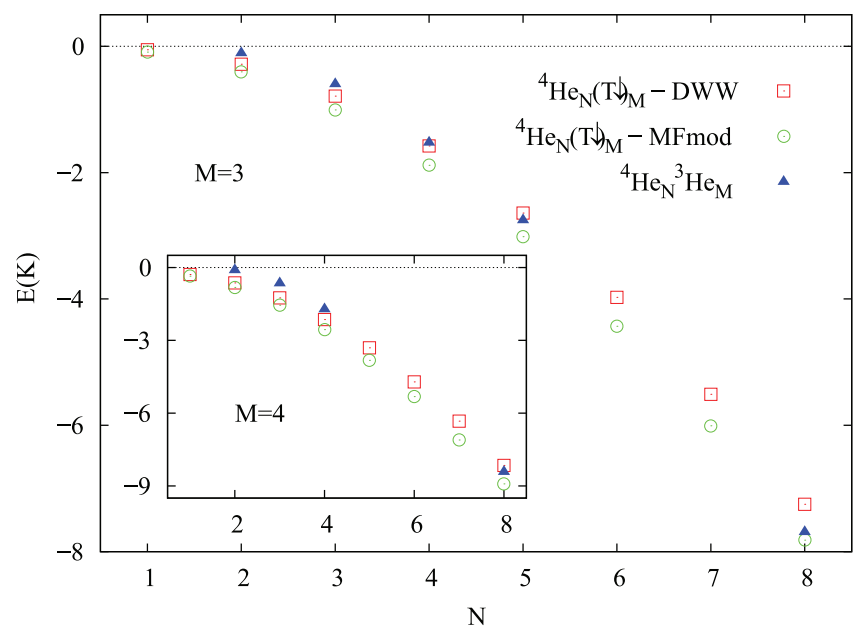

FIG. 4. Comparison of the ground-state energies of ${ }^{4} \mathrm{He}_{N}(\mathrm{~T} \downarrow)_{M}$ clusters for two ${ }^{4} \mathrm{He}-\mathrm{T} \downarrow$ interaction potentials with the ground-state energies of ${ }^{4} \mathrm{He}_{N}{ }^{3} \mathrm{He}_{M}$ clusters for different $N$. The larger figure shows results for $M=3$, while results for $M=4$ are shown in the inset. Axis labels are the same on both figures. Ground-state energies of ${ }^{4} \mathrm{He}_{N}{ }^{3} \mathrm{He}_{M}$ clusters are taken from Ref. 47 and 51.

potentials. As it can be expected, for all the considered potentials two ${ }^{4} \mathrm{He}$ atoms are on average closer than one ${ }^{4} \mathrm{He}$ to a $\mathrm{T} \downarrow$ atom. The average distance between ${ }^{4} \mathrm{He}$ and $\mathrm{T} \downarrow$ atoms is 21.8(5) $\AA$ in case of the MFmod potential, 47(2) $\AA$ for the DWW potential and even 206(15) $\AA$ for the TWW interaction potential. At the same time $<r>$ between ${ }^{4} \mathrm{He}$ atoms is $74(9) \AA$ for the TWW potential. This is even larger than the separation of ${ }^{4} \mathrm{He}$ atoms in a ${ }^{4} \mathrm{He}$ dimer, which for the selected interaction potential is around $50 \AA$. On the other hand, in the case of the MFmod potential $<r>=17.0(4) \AA$ for the ${ }^{4} \mathrm{He}^{-}{ }^{4} \mathrm{He}$ pair (30.4(5) $\AA$ for the DWW potential) which confirms the stability of the cluster. The same floppy behavior can be noticed from the distribution of particle distances to the centre of mass, where in the case of the TWW potential the probability of finding a particle close to the centre of mass is so low that it is not visible in the figure. Correspondingly, the average separation of ${ }^{4} \mathrm{He}$ to the centre of mass is $\left\langle r_{c m}\right\rangle=72(6) \AA$ and $\left\langle r_{c m}\right\rangle=150(11) \AA$ for $\mathrm{T} \downarrow$, in case of TWW potential, which indicates instability of the cluster. However, in the course of the simulation there is no indication that particles are increasing their separation. On the other hand, for the DWW/MFmod potential $<r_{c m}>$ is 20.1(5) $\AA / 10.2(3) \AA$ for ${ }^{4} \mathrm{He}$ and $32(1)$ $\AA / 14.3(4) \AA$ for $\mathrm{T} \downarrow$. Thus, both energetically and from the structure analysis ${ }^{4} \mathrm{He}_{2} \mathrm{~T} \downarrow$ is predicted to be one of the most weakly bound three-atomic clusters.

The differences between the distribution functions for the DWW and MFmod interaction 


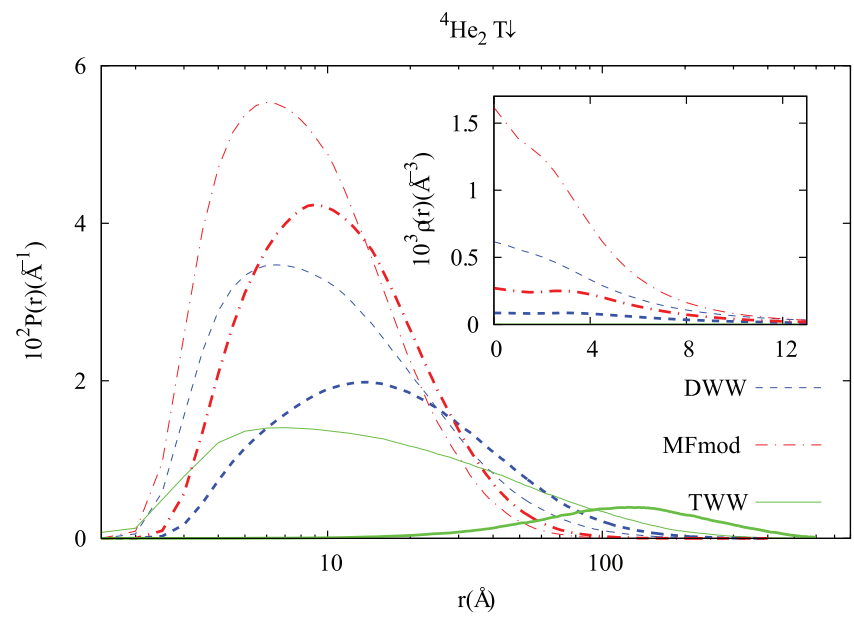

FIG. 5. Distribution of interparticle distances $P(r)$ for three different interaction potentials, where logscale is used in x-axis. Thinner lines correspond to the distribution of ${ }^{4} \mathrm{He}-{ }^{4} \mathrm{He}$ distances, and thicker lines to ${ }^{4} \mathrm{He}-\mathrm{T} \downarrow$ separations. The figure in the inset shows the distributions of the ${ }^{4} \mathrm{He}$ (thinner lines) and $\mathrm{T} \downarrow$ atoms (thicker lines) to the centre of mass $\rho(r)$ for DWW $\underline{39}$ and MFmod $\underline{41}$ potential. The distribution functions for the $\mathrm{TWW}^{42}$ potential are not visible on the picture.

potentials decrease with the increase of the cluster size, but are still noticeable in cases where the number of $\mathrm{T} \downarrow$ is about the same or larger than the number of the ${ }^{4} \mathrm{He}$ atoms. This can be seen in Fig. 6 which presents density distributions with respect to the centre of mass and the ${ }^{4} \mathrm{He}-{ }^{4} \mathrm{He}$ and ${ }^{4} \mathrm{He}-\mathrm{T} \downarrow$ separations distribution for the ${ }^{4} \mathrm{He}_{2}(\mathrm{~T} \downarrow){ }_{4}$ cluster.

Other small mixed clusters, having in particular 4 to 5 atoms are also very floppy, with typical separations between particles of the order of $10 \AA$, for DWW or MFmod potential. Figure 7 shows the particle separation distribution for three small mixed four-particle clusters. As expected, two ${ }^{4} \mathrm{He}$ atoms have on average the smallest separations. The narrowest distributions correspond to the most strongly bound cluster ${ }^{4} \mathrm{He}_{3} \mathrm{~T} \downarrow$. With the exchange of ${ }^{4} \mathrm{He}$ atoms with $\mathrm{T} \downarrow$ ones, clusters become more floppy. It is interesting to notice that in the case of ${ }^{4} \mathrm{He}_{2}(\mathrm{~T} \downarrow)_{2}$ and ${ }^{4} \mathrm{He}(\mathrm{T} \downarrow)_{3}$ clusters the relationship between particle separations distributions for ${ }^{4} \mathrm{He}-\mathrm{T} \downarrow$ and $\mathrm{T} \downarrow-\mathrm{T} \downarrow$ pairs is the opposite.

When $\mathrm{T} \downarrow$ is added to the helium cluster ${ }^{4} \mathrm{He}_{N}$, as a consequence of its smaller mass it tends to stay further away from the centre of mass of the system than ${ }^{4} \mathrm{He}$ atoms, as can be seen in Fig. 8. This is completely analogous to the behavior of ${ }^{3} \mathrm{He}$ in ${ }^{4} \mathrm{He}_{N}{ }^{3} \mathrm{He}$ system. The only exception being that $T \downarrow$ is on average further away from the centre of mass in the ${ }^{4} \mathrm{He}_{N} \mathrm{~T} \downarrow$ cluster than ${ }^{3} \mathrm{He}$ atom in the ${ }^{4} \mathrm{He}_{N}{ }^{3} \mathrm{He}$ cluster, which is a consequence of the weaker 


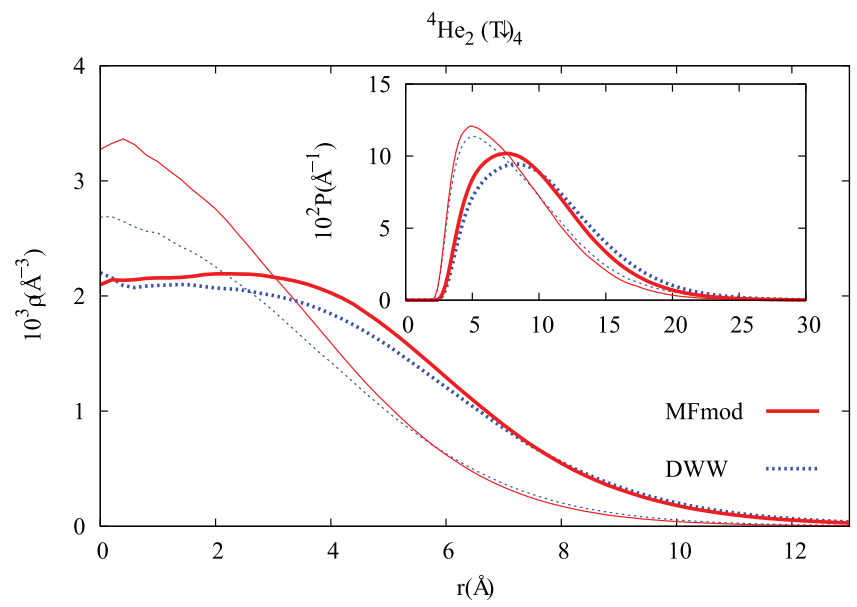

FIG. 6. Distribution of of the ${ }^{4} \mathrm{He}$ (thinner lines) and $\mathrm{T} \downarrow$ atoms (thicker lines) to the centre of mass $\rho(r)$ for $\mathrm{DWW}^{\underline{39}}$ and MFmod $\underline{41}$ potentials. The interparticle distance distribution $P(r)$ is shown on the figure in inset. Thinner lines correspond to the distribution of ${ }^{4} \mathrm{He}^{-}{ }^{4} \mathrm{He}$ distances, and thicker lines to ${ }^{4} \mathrm{He}-\mathrm{T} \downarrow$ separations. $P(r)$ for $\mathrm{T} \downarrow-\mathrm{T} \downarrow$ are very close to $P(r)$ for ${ }^{4} \mathrm{He}-\mathrm{T} \downarrow$ and are thus not shown for better clarity.

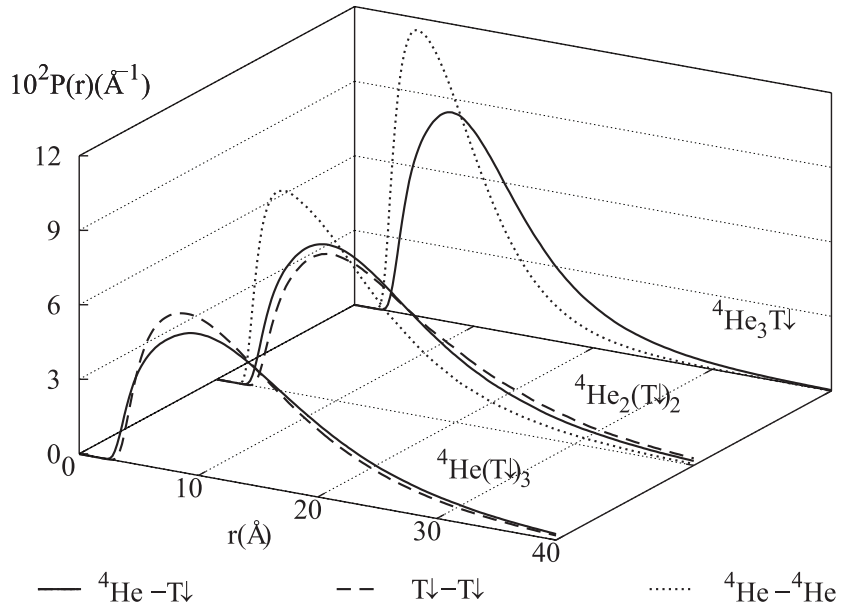

FIG. 7. The distribution of the interparticle distances for three mixed tetramers.

${ }^{4} \mathrm{He}-\mathrm{T} \downarrow$ interaction potential. This can be seen by comparison with the density distribution of ${ }^{3} \mathrm{He}$ in Ref. 52. For $N=3$ there is still appreciable probability for $\mathrm{T} \downarrow$ to be close to the centre of mass, while for $N=8$ this probability is already very low, with $<r_{c m}>=7.33(1) \AA$ and root-mean-square deviation $\Delta r_{c m}=\sqrt{\left\langle r_{c m}^{2}>-<r_{c m}\right\rangle^{2}}$ of $2.2 \AA$. For larger clusters, it is expected that $\mathrm{T} \downarrow$ will be completely pushed to the surface forming the so-called Andreev states, $\stackrel{53}{{ }^{2}}$ like ${ }^{3} \mathrm{He}$ in mixed ${ }^{3} \mathrm{He}-{ }^{4} \mathrm{He}$ clusters. ${ }^{54}$ 


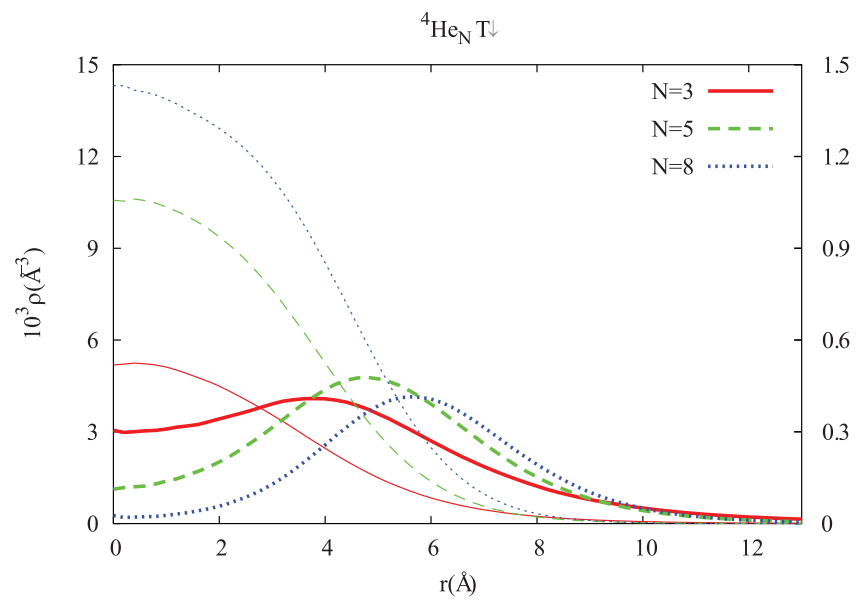

FIG. 8. Density profiles of the ${ }^{4} \mathrm{He}_{N} \mathrm{~T} \downarrow$ system, for $\mathrm{N}=3,5,8$. Thin and thick lines stand for ${ }^{4} \mathrm{He}$ and $\mathrm{T} \downarrow$ atoms, respectively. Left scale is for ${ }^{4} \mathrm{He}$ and the right one for $\mathrm{T} \downarrow$ density distributions.

Having determined the average squared interparticle separations in DMC by pure estimators, it is interesting to investigate if any of the smallest clusters would fit the definition of the quantum halo state. For three-body systems a useful definition has been given in Ref. 27. Quantum halos should obey the condition $\left\langle\rho^{2}\right\rangle / \rho_{o}^{2}>2$, where $\rho$ is the average radial coordinate and $\rho_{0}$ a scaling parameter, defined as

$$
m \rho^{2}=\frac{1}{M} \sum_{i<k} m_{i} m_{k}\left(\boldsymbol{r}_{\boldsymbol{i}}-\boldsymbol{r}_{\boldsymbol{k}}\right)^{2}, \quad m \rho_{0}^{2}=\frac{1}{M} \sum_{i<k} m_{i} m_{k} R_{i k}^{2} .
$$

In the previous equation, $m$ is the arbitrary mass unit, $m_{i}$ is the mass of atom $i, M$ is the total mass and $R_{i k}$ is the two-body scaling length of system $i$ and $k$. For two-body systems $R_{i k}$ can be easily determined as a classical turning point, from $E=V(r)$. In order to make a rough qualitative prediction we here assume that on average each pair contributes with the same amount to the total binding energy. The same assumption has been made in Ref. 29, where clusters of particles of the same type have been considered. Here, this assumption is obviously not fully correct, but we have verified that the final conclusions whether a cluster is a candidate for halo state or not, remain the same if we assume absolute average potential energies of some pairs to be larger and others smaller. Finally, since the classical region is defined by having positive kinetic energy, we estimate the classical radius by equaling the total energy divided by the number of pairs with the corresponding potential energy of each pair. Having thus found all the $R_{i k}$ we calculate the scaling parameter $\rho_{0}$ from Eq. (7). The only bound mixed trimer ${ }^{4} \mathrm{He}_{2}(\mathrm{~T} \downarrow)$ fits the definition of the quantum halo state since 
$\left\langle\rho^{2}\right\rangle / \rho_{o}^{2}=13.6$. The result is insensitive to different types of ${ }^{4} \mathrm{He}-\mathrm{T} \downarrow$ interaction potentials. The authors of Ref. 29 have used the same approach to estimate the halo condition for clusters of four and five particles. If we extend Eq. (17) to larger clusters we find that both ${ }^{4} \mathrm{He}_{2}(\mathrm{~T} \downarrow)_{2}$ and ${ }^{4} \mathrm{He}(\mathrm{T} \downarrow)_{3}$ can be considered quantum halo states in case of the DWW ${ }^{4} \mathrm{He}-\mathrm{T} \downarrow$ interaction potential, since $\left\langle\rho^{2}\right\rangle / \rho_{o}^{2}$ is 2.32 and 2.04 , respectively. This approach is qualitative, but it confirms the extremely weak structure of these systems and motivates their further investigation.

\section{CONCLUSIONS}

The ground state of small mixed clusters composed of helium and spin-polarized tritium has been investigated by quantum Monte Carlo simulations. Significant differences in binding energies are obtained for different ${ }^{4} \mathrm{He}-\mathrm{T} \downarrow$ interaction potentials, especially for the smallest clusters. Nevertheless, the conclusions concerning stability limits are insensitive to these differences, except for the trimer. The mixed dimer does not exist, and the only bound mixed trimer is the ${ }^{4} \mathrm{He}_{2} \mathrm{~T} \downarrow$ one. The latter result is obtained for several ${ }^{4} \mathrm{He}-\mathrm{T} \downarrow$ interaction potentials. However, in the case of the weakest potential (TWW) the cluster ${ }^{4} \mathrm{He}_{2} \mathrm{~T} \downarrow$ is at threshold of separating into a dimer and a free particle and its stability could not be determined with certainty. Due to its large size, this cluster has most of the probability outside the classically allowed regions of space, classifying it as a quantum halo state. Another possible mixed trimer ${ }^{4} \mathrm{He}(\mathrm{T} \downarrow)_{2}$, a candidate for the Borromean state is not bound.

Our results further indicate that two mixed tetramers ${ }^{4} \mathrm{He}_{2}(\mathrm{~T} \downarrow)_{2}$ and ${ }^{4} \mathrm{He}(\mathrm{T} \downarrow){ }_{3}$, could also be considered quantum halos. The largest clusters here analyzed are also very weakly bound and floppy.

\section{ACKNOWLEDGMENTS}

We thank I. Bešlić for useful discussions. J. B. acknowledges support from DGI (Spain) Grant No. FIS2005-04181 and Generalitat de Catalunya Grant No. 2008SGR-04403. P.S. and L.V.M. acknowledge support from MSES (Croatia) under Grant No. 177-1770508-0493. We also acknowledge the support of the Central Computing Services at the Johannes Kepler University in Linz, where part of the computations was performed. In addition, the resources 
of the Isabella cluster at Zagreb University Computing Centre (Srce) and Croatian National Grid Infrastructure (CRO NGI) were used, as well as the resources of the HYBRID cluster at the University of Split, Faculty of Science.

\section{REFERENCES}

${ }^{1}$ W. C. Stwaley and L. H. Nosanow, Phys. Rev. Lett. 36, 910 (1976).

${ }^{2}$ I. Bešlić, L. Vranješ Markić, J. Boronat, Physical Review B 80, 134506 (2009).

${ }^{3}$ M. D. Miller, Phys. Rev. B 18, 4730 (1978).

${ }^{4}$ D. Blume, B. D. Esry, C. H. Greene, N. N. Klausen, G. J. Hanna, Phys. Rev. Lett. 89, $163402(2002)$.

${ }^{5}$ D. V. Fedorov, A. S. Jensen, and K. Riisager, Phys. Rev. C 49, 201 (1994); 50, 2372 (1994).

${ }^{6}$ A. S. Jensen, K. Riisager, D. V. Fedorov, and E. Garrido, Rev. Mod. Phys. 76, 215 (2004).

${ }^{7}$ M. Salci, Sergey B. Levin and Nils Elander, Phys. Rev. A 69, 044501(2004).

${ }^{8}$ I. Bešlić, L. Vranješ Markić, J. Boronat, J. Chem. Phys. 128, 064302 (2008).

${ }^{9}$ I. Bešlić, L. Vranješ Markić, J. Boronat,J. chem. phys. 131, 244506 (2009).

${ }^{10}$ I. Bešlić, L. Vranješ Markić, J. Boronat, Journal of Physics: Conference Series, 150, 032010 (2009).

${ }^{11}$ Y. Li, Q. Gou, and T. Shi, Phys. Rev. A 74, 032502 (2006).

${ }^{12}$ Y. Li and C. D. Lin, J. Phys. B: At. Mol. Opt. Phys. 32, 4877 (1999).

${ }^{13}$ M. Casalegno, M. Mella, G. Morosi and D. Bressanini, J. Chem. Phys. 112, 69 (2000).

${ }^{14}$ F. A. Gianturco, F.Paesani, I, Baccarelli, G. Delgado-Barrio, T. Gonzales-Lezana, S. MiretArtés, P. Villarreal, G. B. Bendazzoli and S. Evangelisti, J. Chem. Phys. 114, 5520 (2001).

${ }^{15}$ Y. D. Liu and P.-N. Roy, J. Chem. Phys. 121, 6282 (2004).

${ }^{16}$ Hiroya Suno, J. Chem. Phys 132, 224311 (2010).

${ }^{17}$ V. Efimov, Phys. Lett. 33B, 563 (1970).

${ }^{18}$ T. Kraemer, M. Mark, P. Waldburger, J. G. Danzl, C. Chin, B. Engeser, A. D. Lange, K. Pilch, A. Jaakkola, H.-C. Ngerl, and R. Grimm, Nature (London) 440, 315 (2006).

${ }^{19}$ T. B. Ottenstein, T. Lompe, M. Kohnen, A. N. Wenz and S. Jochim, Phys. Rev. Lett. 101, 203202 (2008). 
${ }^{20}$ J. H. Huckans, J. R. Williams, E. L. Hazlett, R. W. Stites and K. M. O'Hara, Phys. Rev. Lett. 102, 165302 (2009).

${ }^{21}$ N. Gross, Z. Shotan, S. Kokkelmans and L. Khaykovich, Phys. Rev. Lett. 103, 163202 (2009).

${ }^{22}$ G. Barontini, C. Weber, F. Rabatti, J. Catani, G. Thalhammer, M. Inguscio, F. Minardi Phys. Rev. Lett. 103, 043201 (2009).

${ }^{23}$ M. Zaccanti, B. Deissler, C. D'Errico, M. Fattori, M. Jona-Lasinio, S. Müller, G. Roati, M. Inguscio and G. Modugno, Nature Phys. 5, 586 (2009).

${ }^{24}$ J. von Stecher, J. P. D'Incao and C. H. Greene, Nature Phys. 5, 417-421 (2009).

${ }^{25}$ F. Ferlaino, S. Knoop, M. Berninger, W. Harm, J. P. D'Incao, H. C. Nagerl, R. Grimm, Phys. Rev. Lett. 102, 140401 (2009).

${ }^{26}$ J. von Stecher, J. Phys. B: At. Mol. Opt. Phys. 40, 1 (2010).

${ }^{27}$ A. S. Jensen, K. Riisager, D. V. Fedorov and E. Garrido, Rev. Mod. Phys. 76, 215 (2004).

${ }^{28}$ G. J. Hanna and D. Blume, Phys. Rev. A 74, 063604 (2006).

${ }^{29}$ M. T. Yamashita, D. V. Fedorov and A. S. Jensen, Phys. Rev. A 81, 063607 (2010)

${ }^{30}$ R. A. Aziz, F. R. W. McCourt, and C. C. K. Wong, Mol. Phys. 61, 1487 (1987).

${ }^{31}$ T. Korona, H. L. Williams, R. Bukowski, B. Jeziorski and K. Szalewicz, J. Chem. Phys. 106, 5109 (1997).

${ }^{32}$ W. Kolos and L. Wolniewicz, J. Chem. Phys. 43, 2429 (1965); Chem. Phys. Lett. 24, 457 (1974).

${ }^{33}$ L. Vranješ Markić, J. Boronat and J. Casulleras, Phys. Rev. B 75, 064506 (2007).

${ }^{34}$ M. J. Jamieson, A. Dalgarno, and L. Wolniewicz, Phys. Rev. A 61, 042705 (2000).

${ }^{35}$ Zong-Chao Yan, James F. Babb, A. Dalgarno, and G. W. F. Drake, Phys. Rev A 54, 2824(1996).

${ }^{36}$ W. Kolos and J. Rychlewski, J. Mol. Spectrosc. 143, 237 (1990).

${ }^{37}$ J. H. Freed, J. Chem. Phys. 72, 1414 (1980).

${ }^{38}$ R. Jochemsen, A. J. Berlinsky, and W. N. Hardy, Can. J. Phys. 62, 751759 (1984).

${ }^{39}$ G. Das, A. F. Wagner, and A. C. Wahl, J. Chem. Phys. 68, 4917 (1978).

${ }^{40}$ W. Meyer, L. Frommhold, Theor. Chim. Acta 88, 201 (1994).

${ }^{41}$ H-K.Chung and A. Dalgarno, Phys. Rev. A 66, 012712 (2002).

${ }^{42}$ J. P. Toennies, W. Welz, and G. Wolf, Chem. Phys. Lett. 44, 5 (1976).

${ }^{43}$ E. Krotscheck, R. E. Zillich, Phys. Rev. B 77, 094507 (2008). 
${ }^{44}$ K.T.Tang and X.D.Yang, Phys. Rev. A 42, 311 (1990).

${ }^{45}$ J. Boronat and J. Casulleras, Phys. Rev. B 498920 (1994).

${ }^{46}$ J. Casulleras and J. Boronat, Phys. Rev. B 52, 3654 (1995).

${ }^{47}$ I. Bešlić, L. Vranješ Markić and S. Kilić, J. Low Temp. Phys. 143, 257 (2006).

${ }^{48}$ D. Cvetko, A. Lausi, A. Morgante, F. Tommasini, P. Cortona and M. G. Dondi, J. Chem. Phys. 100, 2052 (1994).

${ }^{49}$ B. D. Esry, C. D. Lin and Chris H. Greene, Phys. Rev A 54, 394 (1996).

${ }^{50}$ A. Kalinin, O. Kornilov, W. Schölkopf and J. P. Toennies, Phys. Rev. Lett. 95, 113402 (2005).

${ }^{51}$ R. Guardiola, J. Navarro, Phys. Rev. A, 68, 055201 (2003).

${ }^{52}$ D. Bressanini, M. Zavaglia, M. Mella and G. Morosi, J. Chem. Phys. 112, 717 (2000).

${ }^{53}$ A. F. Andreev, Sov. Phys. JETP 23, 939 (1966).

${ }^{54}$ M. Barranco, R. Guardiola, S. Hernndez, R. Mayol, and M. Pi, J. Low Temp. Phys. 142, 1 (2006), and references therein. 